\title{
Prognostic Role of S100A8 and S100A9 Protein Expressions in Non-small Cell Carcinoma of the Lung
}

Hyun Min Koh ${ }^{1}$. Hyo Jung $A n^{1}$

Gyung Hyuck $\mathrm{Ko}^{2,3,4}$

Jeong Hee Lee ${ }^{2,3,4} \cdot$ Jong Sil Lee ${ }^{2,3,4}$

Dong Chul Kim ${ }^{2,3,4} \cdot$ Jung Wook Yang ${ }^{4}$

Min Hye Kim ${ }^{4}$. Sung Hwan Kim ${ }^{2,3,5}$

Kyung Nyeo Jeon ${ }^{2,3,6}$

Gyeong-Won Lee ${ }^{2,3,7} \cdot$ Se Min Jang ${ }^{8}$

Dae Hyun Song ${ }^{1,2,3}$

${ }^{1}$ Department of Pathology, Gyeongsang National University Changwon Hospital, Changwon; ${ }^{2}$ Gyeongsang National University School of Medicine, Jinju; ${ }^{3}$ Gyeongsang Institute of Health Science, Jinju; " Department of Pathology, Gyeongsang National University Hospital, Jinju; Departments of ${ }^{5}$ Thoracic and Cardiovascular Surgery and ${ }^{6}$ Radiology, Gyeongsang National University Changwon Hospital, Changwon; ${ }^{7}$ Department of Internal Medicine, Gyeongsang National University Hospital, Jinju;

${ }^{8}$ Department of Pathology, Konyang University Hospital, Daejeon, Korea

Received: May 15, 2018

Revised: November 7, 2018

Accepted: November 12, 2018

Corresponding Author

Dae Hyun Song, MD, PhD

Department of Pathology, Gyeongsang National

University School of Medicine, 79 Gangnam-ro,

Jinju 52727, Korea

Tel: +82-55-214-3150

Fax: $+82-55-214-3174$

E-mail: golgy@hanmail.net
Background: S100A8 and S100A9 have been gaining recognition for modulating tumor growth and metastasis. This study aimed at evaluating the clinical significance of S100A8 and S100A9 in non-small cell lung cancer (NSCLC). Methods: We analyzed the relationship between S100A8 and S100A9 expressions, clinicopathological characteristics, and prognostic significance in tumor cells and peritumoral inflammatory cells. Results: The positive staining of S100A8 in tumor cells was significantly increased in male $(p<.001)$, smoker $(p=.034)$, surgical method other than lobectomy $(p=.024)$, squamous cell carcinoma (SQCC) $(p<.001)$ and higher TNM stage $(p=.022)$ compared with female, non-smoker, lobectomy, adenocarcinoma (ADC), and lower stage. The proportion of tumor cells stained for S100A8 was related to histologic type $(\mathrm{p}<.001)$ and patient $\operatorname{sex}(p=.027)$. The proportion of inflammatory cells stained for S100A8 was correlated with patient age $(p=.022)$, whereas the proportion of inflammatory cells stained for S100A9 was correlated with patient sex $(p<.001)$ and smoking history $(p=.031)$. Moreover, positive staining in tumor cells, more than $50 \%$ of the tumor cells stained and less than $30 \%$ of the inflammatory cells stained for S100A8 and S100A9 suggested a tendency towards increased survivability in SQCC but towards decreased survivability in ADC. Conclusions: S100A8 and S100A9 expressions might be potential prognostic markers in patients with NSCLC.

Key Words: S100A8; S100A9; Carcinoma, non-small cell lung; Prognosis
Lung cancer is a leading cause of cancer-related morbidity and mortality worldwide. ${ }^{1}$ Non-small cell lung cancer (NSCLC) accounts for more than $85 \%$ of all lung cancers and majority of them are suffering from NSCLC at an advanced stage. ${ }^{2}$ Despite the discovery of molecular mutations and advances in diagnosis and treatment, the prognosis for patients with NSCLC remains poor, and a considerable number of patients experience recurrence. ${ }^{1}$ Therefore, discovery of new biomarkers aiding in early detection and accurate prediction of tumor behavior could improve patient survival rate. ${ }^{3}$

S100 proteins are a calcium-binding protein family with at least twenty members. ${ }^{4}$ They have a variety of intracellular and extracellular functions, such as calcium homeostasis, regulation of enzyme activity, protein phosphorylation, cytoskeletal components, transcriptional factors, and chemo-attraction for leukocytes, and macrophage attraction. ${ }^{4,5}$ In addition to their physiological functions, it has been demonstrated that the expression levels of S100 proteins increase in many diseases, including 
cancers. ${ }^{4}$ Recent clinical and experimental data have suggested that changes in the expression and/or function of $\$ 100$ proteins may represent a key step during cancer development. ${ }^{6}$ Moreover, genomic rearrangements at the chromosomal region 1q21, where most of the $S 100$ genes are clustered, were frequently observed in human epithelial tumors, e.g., the lung, breast, esophagus, colorectal, and liver, as well as in tumors of soft tissue and bone. ${ }^{6}$

S100A8 (calgranulin A) and S100A9 (calgranulin B) are members of the $S 100$ protein family, and have been reported to be overexpressed in various kinds of cancers. ${ }^{6}$ Previous studies have suggested that S100A8 and S100A9 play key roles in modulation of inflammation and immune response, which is conducive to genesis of almost all tumors. ${ }^{7}$ And several studies demonstrated that S100A8 and S100A9 promote tumor cell proliferation and invasion, and enhance metastatic process, establishing pre-metastatic niches. ${ }^{8-10}$ Moreover, some authors reported that gene expression profiling of lung tissue at exosome induced pre-metastatic niches that revealed up-regulation of genes involved in extracellular matrix remodeling and inflammation, effectors of pre-metastatic niche formation such as S100A8 and S100A9. ${ }^{11-13}$ However, little is known about the expressions and prognostic significance of S100A8 and S100A9 in lung cancer.

In this study, we aimed to evaluate the expressions of S100A8 and S100A9 and the relationship between S100A8 and S100A9 expressions, clinicopathological characteristics, and prognostic significance in NSCLC. We also analyzed to compare S100A8 and S100A9 expression between peritumoral inflammatory cells and tumor cells.

\section{MATERIALS AND METHODS}

\section{Patients and clinicopathological data}

Samples from 148 patients who underwent surgical resection for NSCLC between January, 2002 and December, 2009 at Gyeongsang National University Hospital (Jinju, Korea) were examined by two pathologists. Stages were determined according to the guidelines in the American Joint Committee on Cancer Tumor Node Metastasis (TNM) Classification of Malignant Tumors, seventh edition. The histologic type and differentiation grade of the tumors were determined using the classification system of the World Health Organization, fourth edition. Clinical data and survival data were collected through medical record examination and National Statistical Office (Seoul, Korea) records. ${ }^{14}$ Disease-free survival (DFS) was defined as the period from the date of surgery to the date of cancer recurrence, and disease-specific survival (DSS) was defined as from the date of surgery to the date of death, which was mostly due to NSCLC. ${ }^{15}$ Smoking history was defined as non-smokers ( $<100$ lifetime cigarettes) or smokers including current smokers and ex-smokers. This study was approved by the Institutional Review Board of Gyeongsang National University Hospital with a waiver of informed consent (2017-03-027).

\section{Tissue microarray construction}

Hematoxylin and eosin-stained slides were reviewed and the most representative tumor area was marked based on its major differentiation and location near the invasive front on formalinfixed, paraffin-embedded tissue blocks. ${ }^{14} \mathrm{~A}$ core sized $3 \mathrm{~mm}$ in diameter was obtained from each case. In total, five tissue microarray (TMA) blocks were constructed with 30 cores in each block.

\section{Immunohistochemistry}

Immunohistochemistry was performed on $4 \mu$ m thick sections from TMA blocks. Tissues were stained with monoclonal antiS100A8 antibody at a dilution of 1:500 (EPR3554, Abcam, Cambridge, UK) and monoclonal anti-S100A9 antibody at a dilution of 1:250 (EPR3555, Abcam) using an automated immunostainer (Benchmark Ultra, Ventana Medical Systems Inc., Tucson, AZ, USA). Lymphoid cells in tonsil served as positive control for S100A8 and S100A9. The primary antibody was omitted for negative control.

\section{S100A8 and S100A9 expressions}

Immunohistochemical staining for S100A8 and S100A9 were evaluated in each TMA sample by visual counting of the tumor cells and peritumoral inflammatory cells that were defined as tumor infiltrating immune cells (Fig. 1). The staining result of tumor cells for S100A8 and S100A9 was determined as negative (not stained) or positive (stained). The proportion of tumor cells stained for S100A8 and S100A9 was categorized as less than $50 \%$ or more than $50 \% .^{16,17}$ We also assessed the proportion of inflammatory cells stained for S100A8 and S100A9 classified as following: less than $30 \%$ or more than $30 \%$. To confirm reproducibility, all samples were scored by two independent pathologists in a blind manner. If discrepancies occurred, a consensus score was reached.

\section{Statistical analysis}

The relationship between categorical variables was determined using chi-square test. The prognostic significance of various clinicopathological characteristics for DFS and DSS was assessed by the Cox proportional hazard regression method. DFS and 


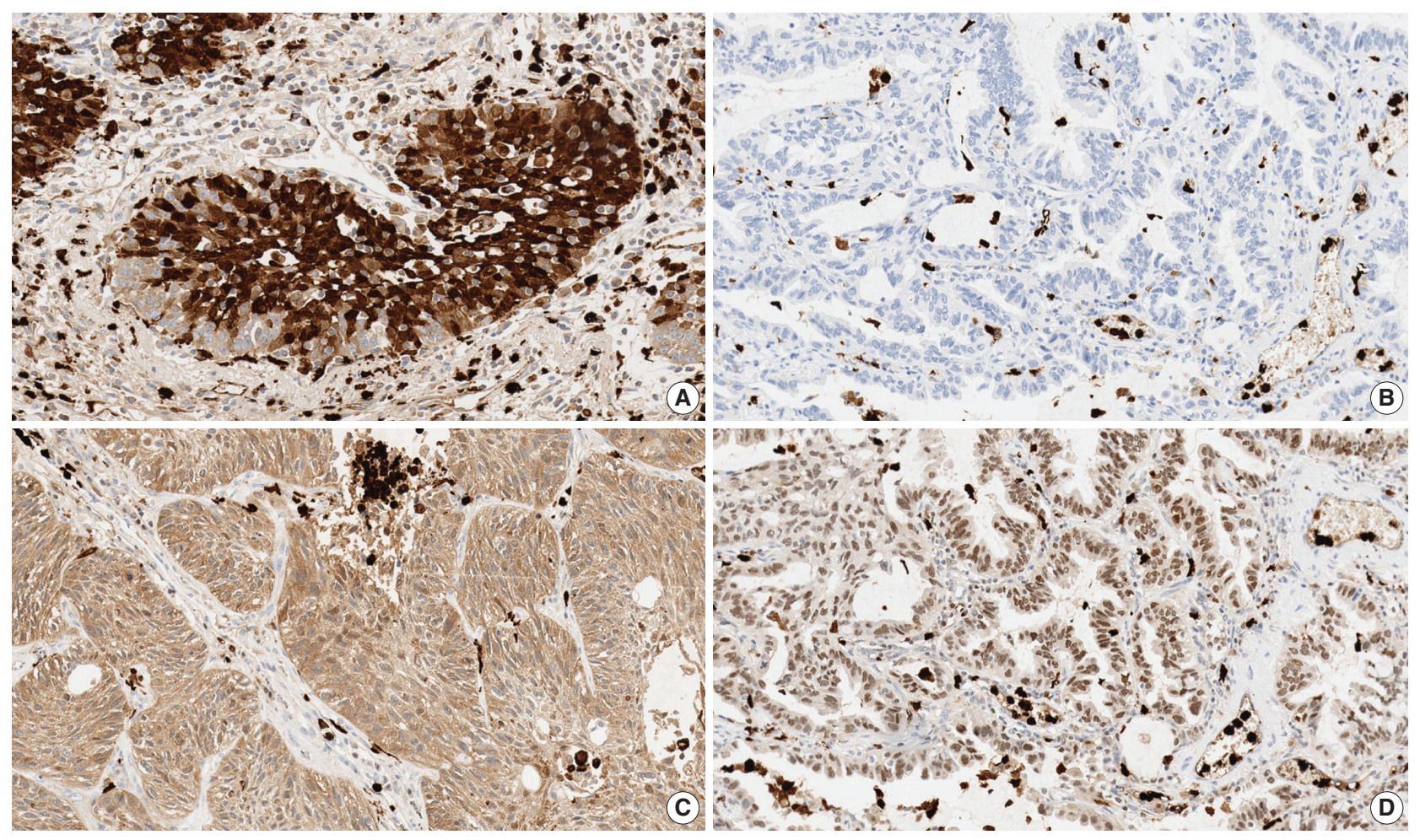

Fig. 1. S100A8 and S100A9 expressions in non-small cell lung cancer. Tumor cells and inflammatory cells stained for S100A8 in squamous cell carcinoma (A), inflammatory cells stained for S100A8 in adenocarcinoma (B), tumor cells and inflammatory cells stained for S100A9 in squamous cell carcinoma (C), tumor cells and inflammatory cells stained for S100A9 in adenocarcinoma (D).

DSS were analyzed using the Kaplan-Meier method with log-rank test between the groups. $\mathrm{p}<.05$ was considered as statistically significant. The analysis was performed using IBM SPSS ver. 25.0 (IBM Corp., Armonk, NY, USA).

\section{RESULTS}

\section{Patient characteristics}

Clinicopathological information of the patients is shown in Table 1. The mean age of the patients was 64.85 years (range, 31 to 77 years). Histologic types of the tumor were as follows: squamous cell carcinoma (SQCC) in 96 (64.9\%), adenocarcinoma (ADC) in 37 (25\%), large cell neuroendocrine carcinoma in eight (5.4\%), and others including pleomorphic and mucoepidermoid carcinoma in seven (4.7\%). The most prevalent histologic feature of SQCC was moderately differentiated in 59 (61.4\%) and ADC was acinar type in 15 (40.5\%). As for the TNM stage, $83(56.1 \%)$ were stage I, 51 (34.5\%) were stage II, $12(8.1 \%)$ were stage III, and two $(1.3 \%)$ were stage IV. Of all the recruited patients, 130 patients (87.9\%) underwent lobectomy including all ADC cases, while the remaining 18 patients (12.1\%) underwent pneumonectomy, bilobectomy, or sleeve lobectomy.
Relationship between S100A8 and S100A9 expressions and clinicopathological characteristics in tumor cells

The relationship between S100A8 and S100A9 expression and clinicopathological characteristics in tumor cells is shown in Table 2. Positive staining for S100A8 was significantly increased in male $(\mathrm{p}<.001)$, smoker $(\mathrm{p}=.034)$, surgical methods other than lobectomy such as bilobectomy or sleeve lobectomy and pneumonectomy ( $\mathrm{p}=.024)$, SQCC $(\mathrm{p}<.001)$ and higher TNM stage $(\mathrm{p}=.022)$ compared with female, non-smoker, lobectomy, $\mathrm{ADC}$ and lower stage. The association between positive staining for S100A8 and surgical method was derived from SQCC cases because lobectomy was done for all ADC cases. The proportion of tumor cells stained for S100A8 showed significant association with histologic type $(\mathrm{p}<.001)$ and patient $\operatorname{sex}(\mathrm{p}=.027)$. However, positive staining for S100A9 and proportion of tumor cells stained for S100A9 did not show any significant correlation with clinicopathological characteristics.

Relationship between proportion of inflammatory cells stained for S100A8 and S100A9 and clinicopathological characteristics

The relationship between proportion of inflammatory cells 
Table 1. Clinicopathological characteristics of the patients

\begin{tabular}{|c|c|}
\hline Characteristic & No. $(\%)(n=148)$ \\
\hline Mean age (yr) & 64.85 \\
\hline Male sex & $125(84.5)$ \\
\hline Smoking history & $97(65.5)$ \\
\hline \multicolumn{2}{|l|}{ Surgical procedure } \\
\hline Lobectomy & $130(87.9)$ \\
\hline Bilobectomy or sleeve lobectomy & $3(2)$ \\
\hline Pneumonectomy & $15(10.1)$ \\
\hline \multicolumn{2}{|l|}{ Histologic type } \\
\hline Squamous cell carcinoma & $96(64.9)$ \\
\hline Well-differentiated & 15 \\
\hline Moderately-differentiated & 59 \\
\hline Poorly- differentiated & 22 \\
\hline Adenocarcinoma & $37(25)$ \\
\hline Acinar & 15 \\
\hline Solid & 6 \\
\hline Papillary & 8 \\
\hline Micropapillary & 3 \\
\hline Lepidic & 3 \\
\hline Mucinous & 2 \\
\hline Large cell neuroendocrine carcinoma & $8(5.4)$ \\
\hline Others & $7(4.7)$ \\
\hline \multicolumn{2}{|l|}{ Tumor-node-metastasis stage } \\
\hline la & $35(23.6)$ \\
\hline $\mathrm{lb}$ & 48 (32.4) \\
\hline Ila & $11(7.4)$ \\
\hline llb & $40(27.0)$ \\
\hline Illa & $4(2.7)$ \\
\hline Illb & $8(5.4)$ \\
\hline IV & $2(1.4)$ \\
\hline Median survival (mo) & 37 \\
\hline Five-year survival rate & $33(22.3)$ \\
\hline
\end{tabular}

stained for S100A8 and S100A9 and clinicopathological characteristics is shown in Table 3 . The proportion of inflammatory cells stained for S100A8 was significantly correlated with patient age ( $\mathrm{p}=.022)$ but not with other factors, and the proportion of inflammatory cells stained for S100A9 showed significant correlation with patient $\operatorname{sex}(\mathrm{p}<.001)$ and smoking history $(\mathrm{p}=.031)$.

\section{Relationship between S100A8 and S100A9 expressions in tumor cells and inflammatory cells}

The relationship between S100A8 and S100A9 expressions in tumor cells and inflammatory cells is shown in Table 4. In tumor cells, the positivity of S100A8 and S100A9 significantly correlated with the proportion of tumor cells stained for each protein $(\mathrm{p}<.001)$. The positivity and proportion of tumor cells stained for S100A8 showed significant association with positivity of $\mathrm{S100A9}$ ( $\mathrm{p}<.001$ ), and the proportion of tumor cells stained for S100A8 showed significant correlation with the proportion of tumor cells stained for S100A9 ( $p<.001)$. In inflammatory cells, the proportion of inflammatory cells stained for S100A8 showed significant correlation with that of inflammatory cells stained for S100A9 ( $\mathrm{p}<.001)$. In addition, the positivity of S100A8 and S100A9 in tumor cells was significantly correlated with the proportion of inflammatory cells stained for $\mathrm{S} 100 \mathrm{~A}$ ) ( $\mathrm{p}=$ .031 and $\mathrm{p}=.010$, respectively).

\section{S100A8 andS100A9 expressions and survival analysis}

The mean follow-up time of the patients in this study was 113 months. In total, $48.6 \%$ of the patients $(\mathrm{n}=72)$ had recurrence and $41.9 \%(\mathrm{n}=62)$ died. The median DSS time was 37 months. The recurrence rate and survival rate had no significant differences between any groups, such as the staining result of tumor cells, proportion of tumor cells and inflammatory cells stained for S100A8 and S100A9 in NSCLC.

In SQCC, $54.2 \%$ of the patients $(\mathrm{n}=52)$ had recurred and $46.9 \%(n=45)$ died. DSS rate was significantly higher in the group in which more than $50 \%$ of the tumor cells were stained for S100A9 ( $n=36,62.1 \%$ ) compared with the group in which less than $50 \%$ of the tumor cells were stained $(\mathrm{n}=15,39.5 \%)(\mathrm{p}=$ .030). Indeed, Kaplan Meier analysis confirmed that more than $50 \%$ of the tumor cells stained for S100A9 were significantly associated with a favorable DSS ( $\mathrm{p}=.016)$. Moreover, a multivariate $\mathrm{Cox}$ proportional hazards regression model demonstrated that more than $50 \%$ of the tumor cells stained for S100A9 represent an independent factor for good DSS (hazard ratio, $0.483 ; 95 \%$ confidence interval, 0.265 to $0.880 ; \mathrm{p}=.017$ ) (Table 5). But DFS and DSS rates did not show statistical differences between the groups with staining results of the tumor cells and the proportion of inflammatory cells stained for S100A8 and S100A9. However, the groups with positive staining in tumor cells for S100A8 and S100A9 and the group with more than $50 \%$ of the tumor cells stained for S100A8 did show a tendency towards increased DFS and DSS rates compared with the groups showing negative staining or less than $50 \%$ of the tumor cells stained. Also, groups showing less than 30\% of the inflammatory cells stained for S100A8 and S100A9 revealed an increasing trend of better prognosis compared with groups showing more than $30 \%$ stained (Fig. 2A-F).

In $A D C, 32.4 \%$ of the patients $(n=12)$ had recurrence and $21.6 \%(n=8)$ died. The DFS and DSS rates had no significant differences between any groups, such as staining result of the tumor cells, the proportion of tumor cells and inflammatory cells stained for S100A8 and S100A9. However, the groups with positive staining in tumor cells and groups with more than $50 \%$ of the tumor cells stained for S100A8 and S100A9 did show a 
Table 2. Relationship between S100A8 and S100A9 expressions and clinicopathological characteristics in tumor cells

\begin{tabular}{|c|c|c|c|c|c|c|c|c|c|c|c|c|}
\hline & \multicolumn{6}{|c|}{ S100A8 expression } & \multicolumn{6}{|c|}{ S100A9 expression } \\
\hline & \multicolumn{3}{|c|}{ Staining result of tumor cells } & \multicolumn{3}{|c|}{ Proportion of tumor cells stained } & \multicolumn{3}{|c|}{ Staining result of tumor cells } & \multicolumn{3}{|c|}{ Proportion of tumor cells stainec } \\
\hline & Negative & Positive & $p$-value & $\leq 50 \%$ & $>50 \%$ & $p$-value & Negative & Positive & $\overline{p \text {-value }}$ & $\leq 50 \%$ & $>50 \%$ & $p$-value \\
\hline Age (yr) & & & .645 & & & .237 & & & .334 & & & .090 \\
\hline$<65$ & $20(32.8)$ & $41(67.2)$ & & $48(77.4)$ & $14(22.6)$ & & $6(9.8)$ & $55(90.2)$ & & $31(50.0)$ & $31(50.0)$ & \\
\hline$\geq 65$ & $31(36.5)$ & $54(63.5)$ & & $59(68.6)$ & $27(31.4)$ & & $13(15.3)$ & $72(84.7)$ & & $31(36)$ & $55(64)$ & \\
\hline Sex & & & $<.001$ & & & .027 & & & .434 & & & .867 \\
\hline Male & 35 (28.2) & $89(71.8)$ & & $86(68.8)$ & $39(31.2)$ & & $15(12.1)$ & $109(87.9)$ & & $52(41.6)$ & $73(58.4)$ & \\
\hline Female & $16(72.7)$ & $6(27.3)$ & & $21(91.3)$ & $2(8.7)$ & & 4 (18.2) & $18(81.8)$ & & $10(43.5)$ & $13(56.5)$ & \\
\hline Smoking & & & .034 & & & .450 & & & .816 & & & .500 \\
\hline Non-smoker & $23(46)$ & $27(54)$ & & $38(76)$ & $12(24)$ & & $7(14)$ & $43(86)$ & & $23(46)$ & $27(54)$ & \\
\hline Smoker & 27 (28.4) & 68 (71.6) & & 68 (70.1) & 29 (29.9) & & $12(12.6)$ & $83(87.4)$ & & 39 (40.2) & $58(59.8)$ & \\
\hline Surgery & & & .024 & & & .090 & & & .315 & & & .783 \\
\hline Lobectomy & 49 (38.3) & 79 (61.7) & & 97 (74.6) & $33(25.4)$ & & $18(14.1)$ & $110(85.9)$ & & 55 (42.3) & 75 (57.7) & \\
\hline Others ${ }^{a}$ & $2(11.1)$ & 16 (88.9) & & 10 (55.6) & $8(44.4)$ & & $1(5.6)$ & $17(94.4)$ & & $7(38.9)$ & $11(61.1)$ & \\
\hline Histologic type & & & $<.001$ & & & $<.001$ & & & .060 & & & .100 \\
\hline SQCC & $22(23.4)$ & 72 (76.6) & & $62(64.6)$ & $34(35.4)$ & & $9(9.6)$ & $85(90.4)$ & & 38 (39.6) & 58 (60.4) & \\
\hline$A D C$ & 26 (70.3) & $11(29.7)$ & & 36 (97.3) & $1(12.5)$ & & $7(18.9)$ & $30(81.1)$ & & 17 (45.9) & $20(54.1)$ & \\
\hline LCN & $3(37.5)$ & $5(62.5)$ & & $7(87.5)$ & $1(12.5)$ & & $3(37.5)$ & $5(62.5)$ & & $6(75)$ & $2(25)$ & \\
\hline Others & 0 & $7(100)$ & & $2(28.6)$ & $5(71.4)$ & & 0 & $7(100)$ & & $1(14.3)$ & $6(85.7)$ & \\
\hline TNM stage & & & .022 & & & .356 & & & .503 & & & .977 \\
\hline la & 19 (57.6) & $14(42.4)$ & & $30(85.7)$ & $5(14.3)$ & & $6(18.2)$ & $27(81.8)$ & & $14(40.0)$ & $21(60.0)$ & \\
\hline $\mathrm{lb}$ & $13(27.1)$ & 35 (72.9) & & $32(66.7)$ & 16 (33.3) & & $7(14.6)$ & $41(85.4)$ & & $22(45.8)$ & $26(54.2)$ & \\
\hline lla & $4(36.4)$ & $7(63.6)$ & & $9(81.8)$ & $2(18.2)$ & & $2(18.2)$ & $9(81.8)$ & & $4(36.4)$ & $7(63.6)$ & \\
\hline$\| \mathrm{lb}$ & $10(25.0)$ & $30(75.0)$ & & $26(65.0)$ & $14(35.0)$ & & $2(5.0)$ & $38(95.0)$ & & $15(37.5)$ & 25 (62.5) & \\
\hline IIla & $1(25.0)$ & $3(75.0)$ & & $3(75.0)$ & $1(25.0)$ & & 0 & $4(100)$ & & $2(50.0)$ & $2(50.0)$ & \\
\hline Illb & $2(25.0)$ & $6(75.0)$ & & $5(62.5)$ & $3(37.5)$ & & $2(25.0)$ & $6(75.0)$ & & $4(50.0)$ & $4(50.0)$ & \\
\hline IV & $2(100)$ & 0 & & $2(100)$ & 0 & & 0 & $2(100)$ & & $1(50.0)$ & $1(50.0)$ & \\
\hline
\end{tabular}

Values are presented as number (\%).

Specimens of two patients were not informative for staining result of tumor cells in S100A8 and S100A9 expressions, due to loss of the specimen. SQCC, squamous cell carcinoma; ADC, adenocarcinoma; LCN, large cell neuroendocrine carcinoma; TNM, tumor-node-metastasis.

aOthers include bilobectomy or sleeve lobectomy and pneumonectomy.

tendency towards decreased DFS and DSS rates compared with groups with negative staining and less than $50 \%$ of the tumor cells stained. Also, groups with less than $30 \%$ of the inflammatory cells stained for S100A8 and S100A9 revealed a trend towards worse prognosis compared with groups with more than $30 \%$ stained (Fig. 3A-F).

\section{DISCUSSION}

S100A8 (MRP8, myeloid-related proteins 8) and S100A9 (MRP9) were originally discovered in myeloid cells. ${ }^{8}$ High levels of S100A8 and S100A9 expressions are characteristic of inflammatory conditions, acting as chemotactic molecules that are constitutively expressed by neutrophils, activated monocytes, macrophages, and myeloid-derived suppressor cells-derived exosomes.' S100A8 and S100A9 are often co-expressed and typically function as a S100A8/A9 heterodimer (calprotectin). ${ }^{9}$ Due to their abundance in inflammatory cells, the main functions of S100A8 or S100A9 have been attributed to their roles in inflammatory diseases.

However, the important roles of S100A8 and S100A9 have been increasingly recognized in modulation of tumor growth and metastasis. ${ }^{10}$ Several studies have indicated that S100A8 and S100A9 promote tumor cell proliferation and invasion via activation of various intracellular signaling pathways, including phosphorylation of mitogen-activated protein kinase in tumor cells and promoting activation of nuclear factor $\mathrm{\kappa b}$; they also enhance metastatic process by mediating pre-metastatic niches as chemo-attractants and decreasing matrix metalloproteinases., ${ }^{50-12}$ Moreover, S100A8 and S100A9 are upregulated in various human cancers such as lung, prostate, colon, gastric, breast, and skin cancer, with abundant expression in neoplastic tumor cells as well as infiltrating immune cells surrounding the tumor. ${ }^{5,13}$

There are only a limited number of studies investigating expressions of S100A8 and S100A9 in lung cancer. In the study by Su et al..${ }^{18}$ up-regulation of these proteins was found in ADC 
Table 3. Relationship between proportion of inflammatory cells stained for S100A8 and S100A9 and clinicopathological characteristics

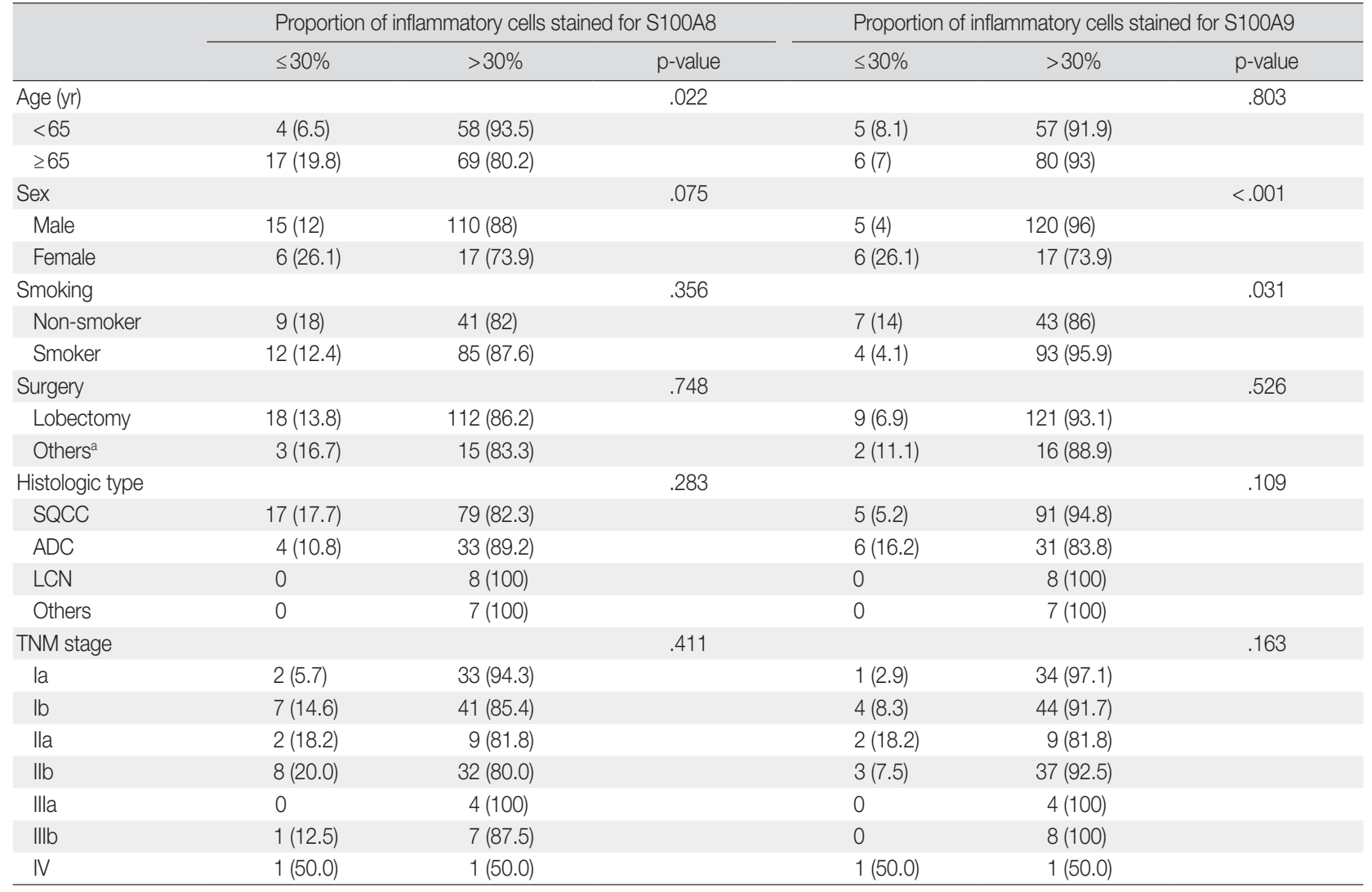

Values are presented as number (\%).

SQCC, squamous cell carcinoma; ADC, adenocarcinoma; LCN, large cell neuroendocrine carcinoma; TNM, tumor-node-metastasis.

aOthers include bilobectomy or sleeve lobectomy and pneumonectomy.

Table 4. The p-values of chi-square tests between S100A8 and S100A9 expressions in tumor cells and inflammatory cells

\begin{tabular}{|c|c|c|c|c|c|c|}
\hline & \multicolumn{4}{|c|}{ Tumor cells } & \multicolumn{2}{|c|}{ Inflammatory cells } \\
\hline & \multicolumn{2}{|c|}{ S100A8 } & \multicolumn{2}{|c|}{ S100A9 } & \multirow{2}{*}{$\begin{array}{c}\text { S100A8 } \\
\text { Proportion }\end{array}$} & \multirow{2}{*}{$\begin{array}{c}\text { S100A9 } \\
\text { Proportion }\end{array}$} \\
\hline & Positivity & Proportion & Positivity & Proportion & & \\
\hline \multicolumn{7}{|l|}{ Tumor cells } \\
\hline \multicolumn{7}{|l|}{ S100A8 } \\
\hline Positivity & - & $<.001$ & $<.001$ & .054 & .050 & .031 \\
\hline Proportion & $<.001$ & - & $<.001$ & $<.001$ & .287 & .083 \\
\hline \multicolumn{7}{|l|}{ S100A9 } \\
\hline Positivity & $<.001$ & $<.001$ & - & $<.001$ & .075 & .010 \\
\hline Proportion & .054 & $<.001$ & $<.001$ & - & .620 & .436 \\
\hline \multicolumn{7}{|l|}{ Inflammatory cells } \\
\hline Proportion for S100A8 & .050 & .287 & .075 & 620 & - & $<.001$ \\
\hline Proportion for S100A9 & .031 & .083 & .010 & .436 & $<.001$ & - \\
\hline
\end{tabular}

that correlated with the clinical characteristics of ADC, inflammation, and stage IV lesions. Arai et al. ${ }^{19}$ reported that the expression level of S100A9 in pulmonary ADC showed a high correlation with poorly differentiated carcinomas, and Huang et al. ${ }^{20}$ exhibited that positive expressions of S100A8 and S100A9 in NSCLC were significantly higher in poorly differentiated tumors compared with moderately- and well-differentiated tumors.

In this study, we found that positive staining of tumor cells for S100A8 correlated significantly with patient sex, smoking history, surgical method, histologic type and TNM stage and the proportion of tumor cells stained for S100A8 was significantly related to the histologic type and patient sex in NSCLC. We also 
Table 5. Cox proportional hazards regression model of disease-free and disease-specific survival for NSCLC patients $(n=133)$

\begin{tabular}{|c|c|c|c|c|c|c|c|c|}
\hline \multirow{3}{*}{ Variable } & \multicolumn{4}{|c|}{ Squamous cell carcinoma } & \multicolumn{4}{|c|}{ Adenocarcinoma } \\
\hline & \multicolumn{2}{|l|}{ DFS } & \multicolumn{2}{|l|}{ DSS } & \multicolumn{2}{|l|}{ DFS } & \multicolumn{2}{|l|}{ DSS } \\
\hline & $\mathrm{HR}(95 \% \mathrm{Cl})$ & $p$-value & $\mathrm{HR}(95 \% \mathrm{Cl})$ & $p$-value & $\mathrm{HR}(95 \% \mathrm{Cl})$ & $p$-value & $\mathrm{HR}(95 \% \mathrm{Cl})$ & $p$-value \\
\hline \multicolumn{9}{|l|}{ Univariate analysis } \\
\hline Age (<65 yr vs $\geq 65$ yr) & $\begin{array}{c}1.402 \\
(0.784-2.506)\end{array}$ & .247 & $\begin{array}{c}1.170 \\
(0.635-2.157)\end{array}$ & .614 & $\begin{array}{c}0.934 \\
(0.296-2.945)\end{array}$ & .934 & $\begin{array}{c}0.818 \\
(0.195-3.428)\end{array}$ & .784 \\
\hline Sex (male vs female) & $\begin{array}{c}0.824 \\
(0.200-3.391)\end{array}$ & .789 & $\begin{array}{c}0.398 \\
(0.055-2.895)\end{array}$ & .363 & $\begin{array}{c}0.736 \\
(0.234-2.320)\end{array}$ & .601 & $\begin{array}{c}0.592 \\
(0.141-2.484)\end{array}$ & .474 \\
\hline $\begin{array}{l}\text { Smoking history } \\
\text { (nonsmoker vs smoker) }\end{array}$ & $\begin{array}{c}0.644 \\
(0.356-1.164)\end{array}$ & .145 & $\begin{array}{c}0.671 \\
(0.355-1.266)\end{array}$ & .218 & $\begin{array}{c}0.918 \\
(0.269-3.139)\end{array}$ & .892 & $\begin{array}{c}1.690 \\
(0.422-6.764)\end{array}$ & .459 \\
\hline $\begin{array}{l}\text { Surgical method } \\
\text { (lobectomy vs others }{ }^{a} \text { ) }\end{array}$ & $\begin{array}{c}1.562 \\
(0.814-2.998)\end{array}$ & .180 & $\begin{array}{c}1.479 \\
(0.730-2.996)\end{array}$ & .278 & $N A^{b}$ & & $N A^{b}$ & \\
\hline TNM stage ( $<$ llb vs $\geq 1 \mathrm{llb})$ & $\begin{array}{c}1.711 \\
(0.984-2.975)\end{array}$ & .057 & $\begin{array}{c}1.966 \\
(1.087-3.558)\end{array}$ & .025 & $\begin{array}{c}2.727 \\
(0.867-8.571)\end{array}$ & .086 & $\begin{array}{c}2.961 \\
(0.738-11.871)\end{array}$ & .126 \\
\hline $\begin{array}{l}\text { Proportion of tumor cells stained } \\
\text { for S100A9 ( } \leq 50 \% \text { vs }>50 \%)\end{array}$ & $\begin{array}{c}0.709 \\
(0.408-1.231)\end{array}$ & .222 & $\begin{array}{c}0.491 \\
(0.272-0.889)\end{array}$ & .019 & $\begin{array}{c}1.625 \\
(0.475-5.557)\end{array}$ & .439 & $\begin{array}{c}2.891 \\
(0.583-14.337)\end{array}$ & .194 \\
\hline \multicolumn{9}{|l|}{ Multivariate analysis } \\
\hline Age (<65 yr vs $\geq 65 \mathrm{yr}$ ) & - & - & - & - & - & - & - & - \\
\hline Sex (male vs female) & - & - & - & - & - & - & - & - \\
\hline Smoking history (nonsmoker vs smoker) & - & - & - & - & - & - & - & - \\
\hline Surgical method (lobectomy vs othersa) & - & - & - & - & - & - & - & - \\
\hline TNM stage (<llb vs $\geq \| l b)$ & $\begin{array}{c}1.736 \\
(0.998-3.022)\end{array}$ & .051 & $\begin{array}{c}2.012 \\
(1.110-3.644)\end{array}$ & .021 & $\begin{array}{c}2.577 \\
(0.805-8.253)\end{array}$ & .111 & $\begin{array}{c}2.528 \\
(0.618-10.336)\end{array}$ & .197 \\
\hline $\begin{array}{l}\text { Proportion of tumor cells stained } \\
\text { for S100A9 ( } \leq 50 \% \text { vs }>50 \%)\end{array}$ & $\begin{array}{c}0.702 \\
(0.402-1.224)\end{array}$ & .212 & $\begin{array}{c}0.483 \\
(0.265-0.880)\end{array}$ & .017 & $\begin{array}{c}1.374 \\
(0.393-4.807)\end{array}$ & .619 & $\begin{array}{c}2.457 \\
(0.484-12.484)\end{array}$ & .278 \\
\hline
\end{tabular}

NSCLC, non-small cell lung cancer; DFS, disease-free survival; DSS, disease-specific survival; HR, hazard ratio; Cl, confidence interval; NA, not applicable; TNM, tumor-node-metastasis.

aOthers include bilobectomy or sleeve lobectomy and pneumonectomy; ${ }^{\text {b}} \mathrm{All}$ of the adenocarcinoma cases underwent lobectomy.

observed that the proportion of inflammatory cells stained for S100A8 was significantly related to the patient age, and that of S100A9 showed significant correlation with patient sex and smoking history in NSCLC. In addition, positivity of tumor cells and/or proportion of tumor cells stained for S100A8 and S100A9 significantly correlated with positivity of tumor cells and/or proportion of inflammatory cells stained for S100A8 and/or S100A9 in NSCLC, indicating that inflammation is closely associated with cancer. ${ }^{21}$ Moreover, we analyzed that DSS rate was significantly higher in the group with more than $50 \%$ of the tumor cells stained for S100A9 compared with the group with less than $50 \%$ stained in SQCC, confirmed by Kaplan-Meier analysis and the multivariate Cox proportional hazards regression model.

Another interesting finding of this study is that the groups with positive staining in tumor cells, the groups with more than $50 \%$ of the tumor cells stained and the groups with less than $30 \%$ of the inflammatory cells stained for S100A8 and S100A9 showed a tendency towards increased DFS and DSS rates in SQCC but towards decreased rates in ADC.

The present study is the first evaluate the expressions of S100A8 and S100A9 in NSCLC and their prognostic significance.

In SQCC, several studies have demonstrated that high expres- sion of S100A8 and/or S100A9 could be a favorable factor while showing frequent loss or reduction of the protein expressions in the head and neck, esophagus and cervix. ${ }^{22-25}$ There are a few studies reporting that S100A8 and S100A9 are related to the regulation of keratinocyte proliferation and differentiation. ${ }^{25,26}$ Our results also reveal that positive staining for S100A8 and S100A9 may have a favorable effect on the prognosis in pulmonary SQCC.

In previous studies, strong S100A8 and S100A9 up-regulation was found in breast, gastric, colorectal, pancreatic, and prostate cancer. The altered S100A8 and/or S100A9 expressions in carcinomas of glandular cell origin, such as breast, lung, and thyroid gland, were related to poor tumor differentiation and more aggressive behavior, which is consistent with our results in ADC. .,17,19,27-33 $^{-1}$

Some studies have shown that cancer-related inflammation is often considered to support malignant progression and metastasis, showing up-regulation of S100A8 and S100A9 by inflammatorycells. ${ }^{910,21,25}$ However, the same inflammatory cells also participate in anti-tumor immunity and restraint of malignant disease. $^{34}$ The balance between anti-tumor and pro-tumor functions can be dependent on polarization state, interaction with tumor microenvironment, and/or the tumor type. ${ }^{10,35}$ In the same 

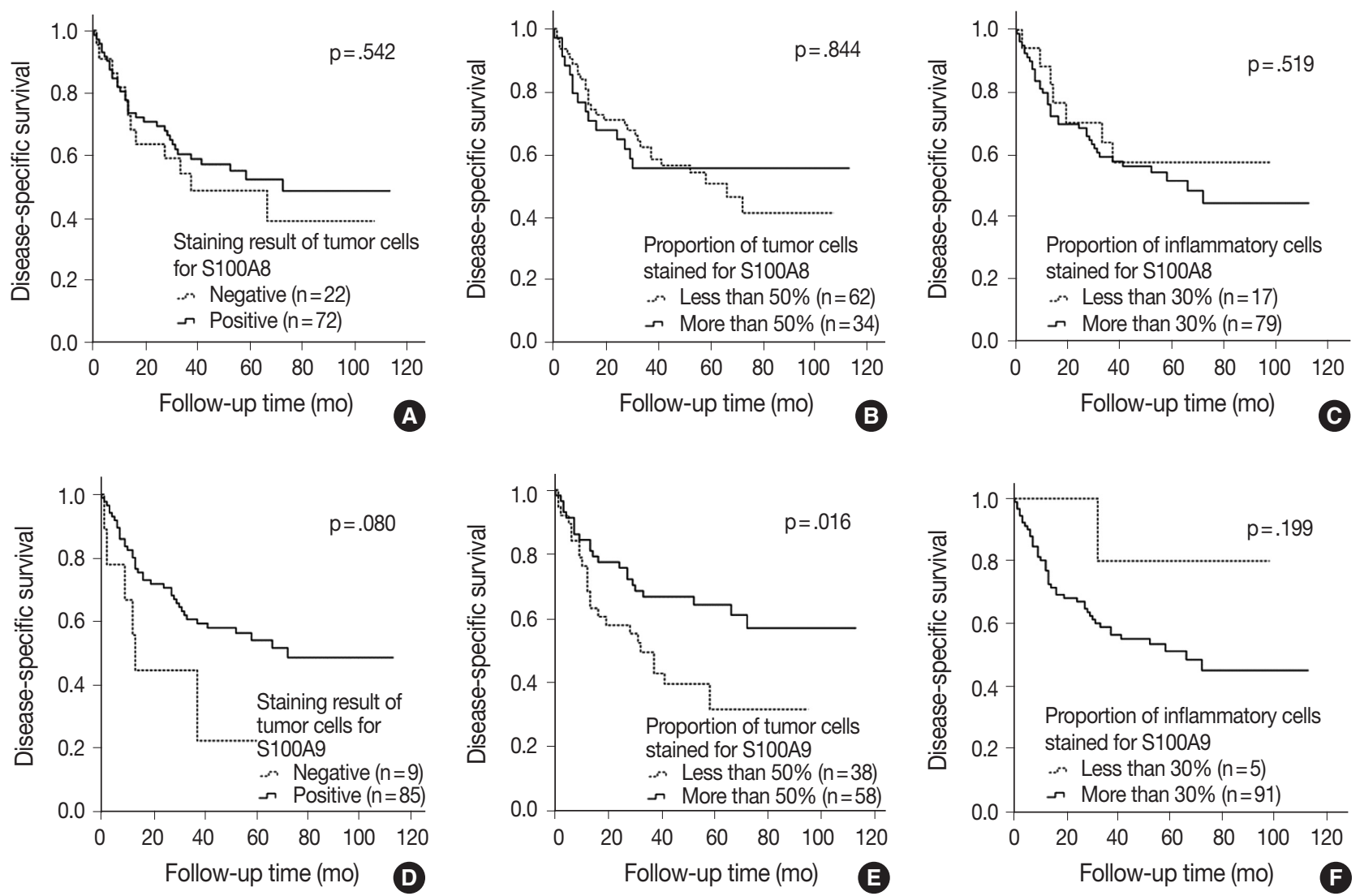

Fig. 2. Kaplan-Meier survival analysis of survival curves based on S100A8 and S100A9 expressions in patients with squamous cell carcinoma. The groups with positive staining in tumor cells $(A, D)$, the groups with more than $50 \%$ of the tumor cells $(B, E)$ stained and the groups with less than 30\% of the inflammatory cells (C, F) stained for S100A8 and S100A9 reveal a tendency towards increased survivability compared with the groups showing negative staining, less than $50 \%$ of the tumor cells stained and more than $30 \%$ of the inflammatory cells stained.

context, our studies reveal that the proportion of inflammatory cells stained for S100A8 and S100A9 may have an influence that is quite opposite in SQCC and ADC.

There are some limitations to this study. For example, we included a TMA core per case in analysis, which could induce a lack of representativeness. In addition, we did not use an adjustment method in scoring of inflammatory cells although tumor infiltrating immune cells consist of various cells. Moreover, the number of ADC cases were small, and they were all removed by lobectomy so we could not use the variable of surgical method in Cox proportional hazards regression model. A large-scale study with an organized study design including a sufficient number of cases and tissue samples is recommended in future studies.

In summary, we have found that positive staining in tumor cells, more than $50 \%$ of the tumor cells stained and less than $30 \%$ of the inflammatory cells stained for S100A8 and S100A9 suggest a tendency towards increased survivability in SQCC, whereas positive staining in tumor cells, more than $50 \%$ of the tumor cells stained and less than $30 \%$ of the inflammatory cells stained for S100A8 and S100A9 suggest a tendency towards decreased survivability in ADC. Consequently, S100A8 and S100A9 expressions could be potential prognostic factors in patients with NSCLC.

Many recent studies have provided evidence that implicates S100A8 and S100A9 to play important roles in cancer. However, the expressions and/or functions of S100A8 and S100A9 are much more complicated, supporting our data. Further studies are needed to fully comprehend their roles in cancer.

\section{ORCID}

Hyun Min Koh: https://orcid.org/0000-0002-7457-7174

Hyo Jung An: https://orcid.org/0000-0002-2068-8370

Gyung Hyuck Ko: https://orcid.org/0000-0002-6721-1828

Jeong Hee Lee: https://orcid.org/0000-0001-5833-4773

Jong Sil Lee: https://orcid.org/0000-0001-9159-7575

Dong Chul Kim: https://orcid.org/0000-0002-9484-7792

Jung Wook Yang: https://orcid.org/0000-0002-9698-3667 

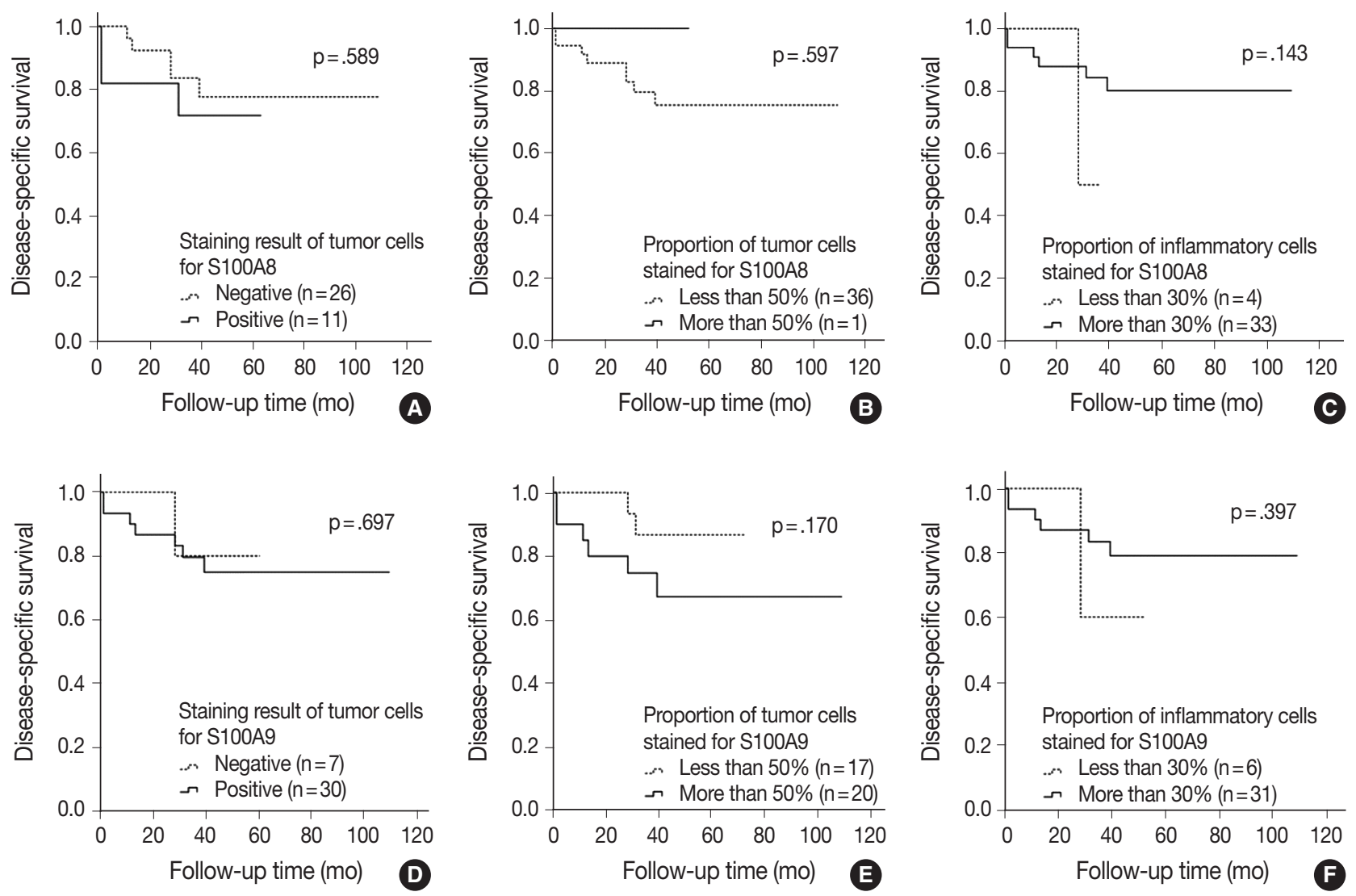

Fig. 3. Kaplan-Meier survival analysis of survival curves based on S100A8 and S100A9 expressions in patients with adenocarcinoma. The groups with positive staining in tumor cells (A, D), the groups with more than $50 \%$ of the tumor cells $(B, E)$ stained and the groups with less than 30\% of the inflammatory cells (C, F) stained for S100A8 and S100A9 reveal a trend towards decreased survivability compared with the groups showing negative staining, less than $50 \%$ of the tumor cells stained and more than $30 \%$ of the inflammatory cells stained.

Min Hye Kim: https://orcid.org/0000-0002-8631-5104

Sung Hwan Kim: https://orcid.org/0000-0002-6888-774X

Kyung Nyeo Jeon: https://orcid.org/0000-0003-2267-0366

Gyeong-Won Lee: https://orcid.org/0000-0002-1781-2262

Se Min Jang: https://orcid.org/0000-0002-5442-3303

Dae Hyun Song: https://orcid.org/0000-0001-7163-0403

\section{Conflicts of Interest}

The authors declare that they have no potential conflicts of interest.

\section{Acknowledgments}

This research was supported by the Basic Science Research Program through the National Research Foundation of Korea (NRF) funded by the Ministry of Education (2017R1C1B5014837).

\section{REFERENCES}

1. Jo YM, Park TI, Lee HY, Jeong JY, Lee WK. Prognostic significance of aquaporin 5 expression in non-small cell lung cancer. J Pathol Transl Med 2016; 50: 122-8.

2. Lee SH, Kim WS, Choi YD, et al. Analysis of mutations in epidermal growth factor receptor gene in Korean patients with non-small cell lung cancer: summary of a nationwide survey. J Pathol Transl Med 2015; 49: 481-8.

3. Fan B, Zhang LH, Jia YN, et al. Presence of S100A9-positive inflammatory cells in cancer tissues correlates with an early stage cancer and a better prognosis in patients with gastric cancer. BMC Cancer 2012; 12: 316.

4. Yasar O, Akcay T, Obek C, Turegun FA. Significance of S100A8, S100A9 and calprotectin levels in bladder cancer. Scand J Clin Lab Invest 2017; 77: 437-41.

5. Salama I, Malone PS, Mihaimeed F, Jones JL. A review of the S100 proteins in cancer. Eur J Surg Oncol 2008; 34: 357-64.

6. Gebhardt C, Nemeth J, Angel P, Hess J. S100A8 and S100A9 in inflammation and cancer. Biochem Pharmacol 2006; 72: 1622-31.

7. Lang B, Shang C, Meng L. Targeted silencing of S100A8 gene by miR-24 to increase chemotherapy sensitivity of endometrial carci- 
noma cells to paclitaxel. Med Sci Monit 2016; 22: 1953-8.

8. Srikrishna G. S100A8 and S100A9: new insights into their roles in malignancy. J Innate Immun 2012; 4: 31-40.

9. Hiratsuka S, Watanabe A, Aburatani H, Maru Y. Tumour-mediated upregulation of chemoattractants and recruitment of myeloid cells predetermines lung metastasis. Nat Cell Biol 2006; 8: 1369-75.

10. Lim SY, Yuzhalin AE, Gordon-Weeks AN, Muschel RJ. Tumor-infiltrating monocytes/macrophages promote tumor invasion and migration by upregulating S100A8 and S100A9 expression in cancer cells. Oncogene 2016; 35: 5735-45.

11. Hiratsuka S, Watanabe A, Sakurai Y, et al. The S100A8-serum amyloid A3-TLR4 paracrine cascade establishes a pre-metastatic phase. Nat Cell Biol 2008; 10: 1349-55.

12. Weidle UH, Birzele F, Kollmorgen G, Rüger R. The multiple roles of exosomes in metastasis. Cancer Genomics Proteomics 2017; 14: 1-15.

13. Armstrong D, Wildman DE. Extracellular vesicles and the promise of continuous liquid biopsies. J Pathol Transl Med 2018; 52: 1-8.

14. Song DH, Ko GH, Lee JH, et al. Myoferlin expression in non-small cell lung cancer: prognostic role and correlation with VEGFR-2 expression. Oncol Lett 2016; 11: 998-1006.

15. Song DH, Ko GH, Lee JH, et al. Prognostic role of myoferlin expression in patients with clear cell renal cell carcinoma. Oncotarget 2017; 8: 89033-9.

16. Choi DK, Li ZJ, Chang IK, et al. Clinicopathological roles of S100A8 and S100A9 in cutaneous squamous cell carcinoma in vivo and in vitro. Arch Dermatol Res 2014; 306: 489-96.

17. Arai K, Takano S, Teratani T, Ito Y, Yamada T, Nozawa R. S100A8 and S100A9 overexpression is associated with poor pathological parameters in invasive ductal carcinoma of the breast. Curr Cancer Drug Targets 2008; 8: 243-52.

18. Su YJ, Xu F, Yu JP, Yue DS, Ren XB, Wang CL. Up-regulation of the expression of S100A8 and S100A9 in lung adenocarcinoma and its correlation with inflammation and other clinical features. Chin Med J (Engl) 2010; 123: 2215-20.

19. Arai K, Teratani T, Nozawa R, Yamada T. Immunohistochemical investigation of S100A9 expression in pulmonary adenocarcinoma: S100A9 expression is associated with tumor differentiation. Oncol Rep 2001; 8: 591-6.

20. Huang H, Huang Q, Tang T, et al. Clinical significance of calciumbinding protein S100A8 and S100A9 expression in non-small cell lung cancer. Thorac Cancer 2018; 9: 800-4.

21. Zhang X, Ai F, Li X, et al. Inflammation-induced S100A8 activates Id3 and promotes colorectal tumorigenesis. Int J Cancer 2015; 137: 2803-14.

22. Zhu H, Wu TC, Chen WQ, et al. Roles of galectin-7 and S100A9 in cervical squamous carcinoma: clinicopathological and in vitro evi- dence. Int J Cancer 2013; 132: 1051-9.

23. Roesch Ely M, Nees M, Karsai S, et al. Transcript and proteome analysis reveals reduced expression of calgranulins in head and neck squamous cell carcinoma. Eur J Cell Biol 2005; 84: 431-44.

24. Kong JP, Ding F, Zhou CN, et al. Loss of myeloid-related proteins 8 and myeloid-related proteins 14 expression in human esophageal squamous cell carcinoma correlates with poor differentiation. World J Gastroenterol 2004; 10: 1093-7.

25. Funk S, Mark R, Bayo P, et al. High S100A8 and S100A12 protein expression is a favorable prognostic factor for survival of oropharyngeal squamous cell carcinoma. Int J Cancer 2015; 136: 2037-46.

26. Voss A, Bode G, Sopalla C, et al. Expression of S100A8/A9 in $\mathrm{HaCaT}$ keratinocytes alters the rate of cell proliferation and differentiation. FEBS Lett 2011; 585: 440-6.

27. El-Rifai W, Moskaluk CA, Abdrabbo MK, et al. Gastric cancers overexpress S100A calcium-binding proteins. Cancer Res 2002; 62: 6823-6.

28. Seth A, Kitching R, Landberg G, Xu J, Zubovits J, Burger AM. Gene expression profiling of ductal carcinomas in situ and invasive breast tumors. Anticancer Res 2003; 23: 2043-51.

29. Stulík J, Osterreicher J, Koupilová K, et al. The analysis of S100A9 and S100A8 expression in matched sets of macroscopically normal colon mucosa and colorectal carcinoma: the S100A9 and S100A8 positive cells underlie and invade tumor mass. Electrophoresis 1999; 20: 1047-54.

30. Shen J, Person MD, Zhu J, Abbruzzese JL, Li D. Protein expression profiles in pancreatic adenocarcinoma compared with normal pancreatic tissue and tissue affected by pancreatitis as detected by twodimensional gel electrophoresis and mass spectrometry. Cancer Res 2004; 64: 9018-26.

31. Hermani A, Hess J, De Servi B, et al. Calcium-binding proteins S100A8 and S100A9 as novel diagnostic markers in human prostate cancer. Clin Cancer Res 2005; 11: 5146-52.

32. Arai K, Teratani T, Kuruto-Niwa R, Yamada T, Nozawa R. S100A9 expression in invasive ductal carcinoma of the breast: S100A9 expression in adenocarcinoma is closely associated with poor tumour differentiation. Eur J Cancer 2004; 40: 1179-87.

33. Ito Y, Arai K, Ryushi, et al. S100A9 expression is significantly linked to dedifferentiation of thyroid carcinoma. Pathol Res Pract 2005; 201: 551-6.

34. DeNardo DG, Andreu P, Coussens LM. Interactions between lymphocytes and myeloid cells regulate pro- versus anti-tumor immunity. Cancer Metastasis Rev 2010; 29: 309-16.

35. Gabrilovich DI, Ostrand-Rosenberg S, Bronte V. Coordinated regulation of myeloid cells by tumours. Nat Rev Immunol 2012; 12: 253-68. 